Physica A 176 (1991) 1-15

North-Holland

INTRODUCTORY LECTURE

\title{
ORDERING IN SUPRAMOLECULAR FLUIDS
}

\section{H.N.W. LEKKERKERKER}

Van't Hoff Laboratorium, Rijksuniversiteit Utrecht, P.O. Box 80051. 3508 TB Utrecht, The Netherlands

\section{Introduction}

The systems that are of interest in this workshop are liquids containing supramolecular objects (colloidal particles, macromolecules, aggregates of surface active agents (surfactants)) with length scales intermediate between the molecular and the macroscopic, say between $10 \mathrm{~nm}$ and $1000 \mathrm{~nm}$. With the appropriate change of scale the thermodynamic and structural properties of such systems may be studied by the traditional theoretical and experimental techniques of condensed matter physics by considering the supramolecular objects as "supramolecules" dispersed in a continuous (but fluctuating) background. For this reason we refer to these systems as supramolecular fluids. In fig. 1 they are classified as intermediate between atomic and molecular liquids on the one hand and granular fluids on the other hand.

The supramolecular objects that will play a role in this workshop can be divided into

(a) rigid colloidal particles,

(b) self- and mutual avoiding semiflexible objects.

Shapes and examples of such objects are indicated in fig. 2 .

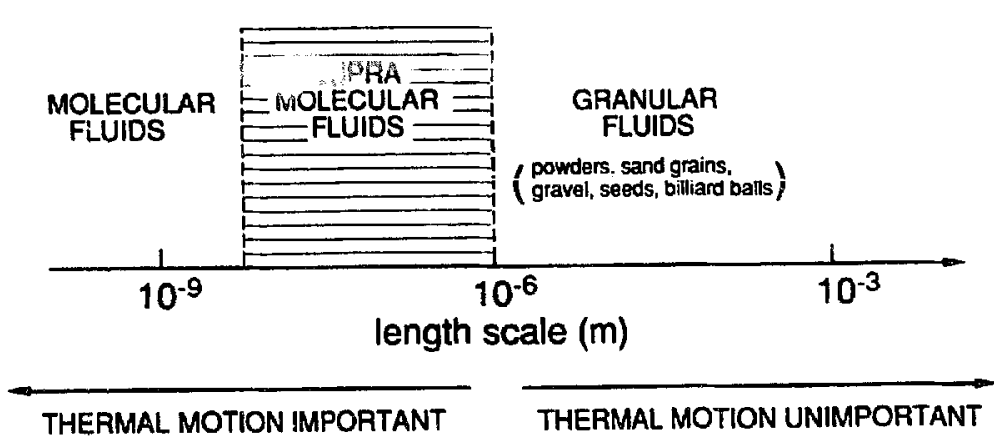

Fig. 1. Classification of supramolecular fluids. 


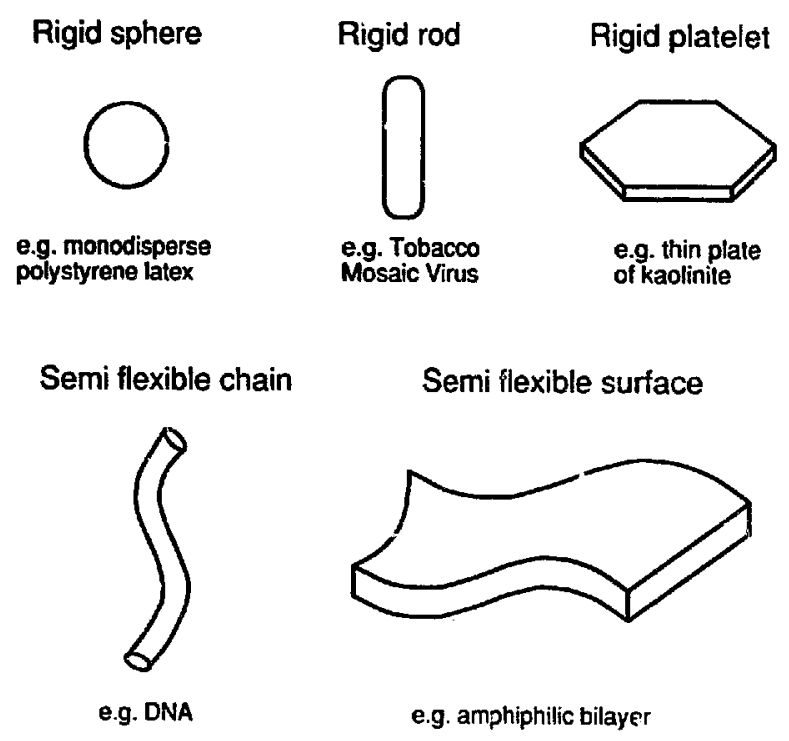

Fig. 2. Shapes and examples of supramolecular objects.

As we will see, the ordering phenomena in supramolecular fluids are primarily dictated by geometry, excluded volume and fiexibility of the supramolecular objects. This is reflected in the common features of the theoretical approaches to treating these systems.

\section{Disorder to order transitions in dispersions of spherical coiloidal particles}

Nowadays one can prepare (almost) monodisperse, spherical colloidal particles whose short-ranged harshly repulsive interparticle forces closely approximate that of hard spheres [1,2]. Dispersions of such colloidal particles can therefore be modeled as supramolecular hard sphere fluids. Computer simulations have made it abundantly clear that the short ranged repulsive intermolecular forces play a dominant role in determining the structural arrangements of molecules in condensed phases. This had led to the use of hard sphere fluids a a basic model in the dcvelopment of theories for the liquid and solid states.

One of the most remarkable features of a hard sphere fluid first revealed by computer simulations $[3,4]$ is the fluid to crystal transition when the number density of the spheres is increased. This transition is pictorially represented in fig. 3 and a schematic representation of the pressure-density phase diagram of the hard sphere fluid is given in fig. 4. 
In recent years the nature of this transition has been clarified considerably by the application of density functional theory [5]. In the density functional approach the non-uniform average density of the solid (characterized by peaks centered at the lattice sites) is treated as if it were the density of an inhomogeneous liquid. The fluid-solid transition can now be viewed as being due to a competition between two terms in the entropy: The long range order reduces the ideal entropy of the solid phase but on the other hand the ordered phase has more free volume which leads to a gain in packing entropy. The first effect is proportional to the number of particles $N$ whereas the second effect is proportional to $N^{2} / V+$ higher terms and thus at high densities the latter effect is dominant leading to a stabilization of the ordered (solid) phase. In his contribution Baus reviews the present status of density functional theory applied to freezing.

As mentioned above, certain colloidal dispersions can be modeled as (supramolecular) hard sphere fluids. Computer simulation and analytical results for hard sphere fluids can therefore directly applied to such colloidal dispersions. In their contribution Pusey and coworkers demonstrate the remarkable power of this idea in analyzing the fluid, crystal and glass states of essentially hard spherical colloidal particles.

Apart from providing a physical example of a hard sphere fluid colloidal dispersions off 2 r unique possibilities to investigate the influence of the range and strength of interparticle interactions. By suitable modification of the

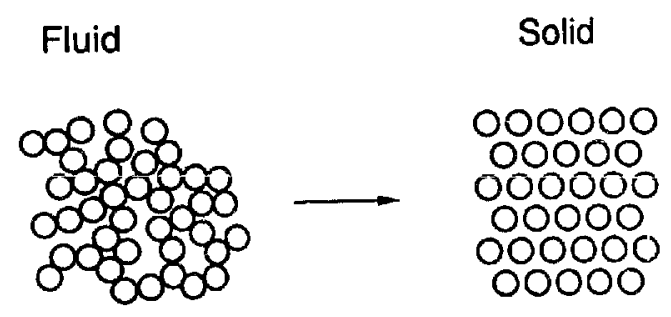

Fig. 3.

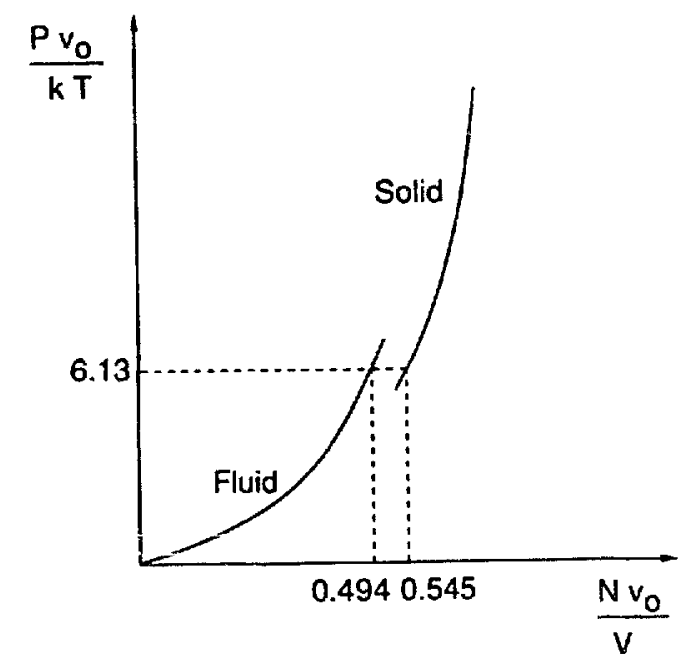

Fig. 4.

Fig. 3. Schematic representation of the fluid to solid transition in a system of hard spheres.

Fig. 4. Pressure-density phase diagram of a system of hard spheres with volume $v_{n}$. 
surface properties of the particles the repulsive interaction can be varied more or less continuously. A particular ingenious method to manipulate the interaction between coiloidal particles has been devised by Skjeltorp. A dispersion of micronsized latex spheres suspended in a ferrofluid is put in an external magnetic field. The holes created by the latex spheres appear to possess magnetic moments antiparallel to the external field. The dipolar interactions between these magnetic moments are controlled by external fields. This gives rise to spectacular effects.

Generally the microstructure in colloidal systems can be markedly influenced by weak external effects. This also applies to the crystallization process in colloidal dispersions. Particularly shear flow has a pronounced effect on the ordering phenomena in colloidal dispersions as is clear from the work of Ackerson [6]. A particularly intriguing observation is that oscillatory shear flow can induce crystal order in a suspension which has an equilibrium liquid-like order.

\section{Liquid crystal phases in dispersions of rigid rod-like dispersions}

That dispersions of rod-like colloidal particles undergo an isotropic-nematic phase transition (pictorially represented in fig. 5) at remarkably low concentrations (volume fractions of about two per cent) was discovered by Zocher [7] in 1925. The explanation for this phenomenon was first given by Onsager [8] in the forties. In particular he showed why dispersions of rigid rod-like particles with purely repulsive interaction: undergo this transition upon increasing the concentration of the particles. The argument is in fact similar to the one that
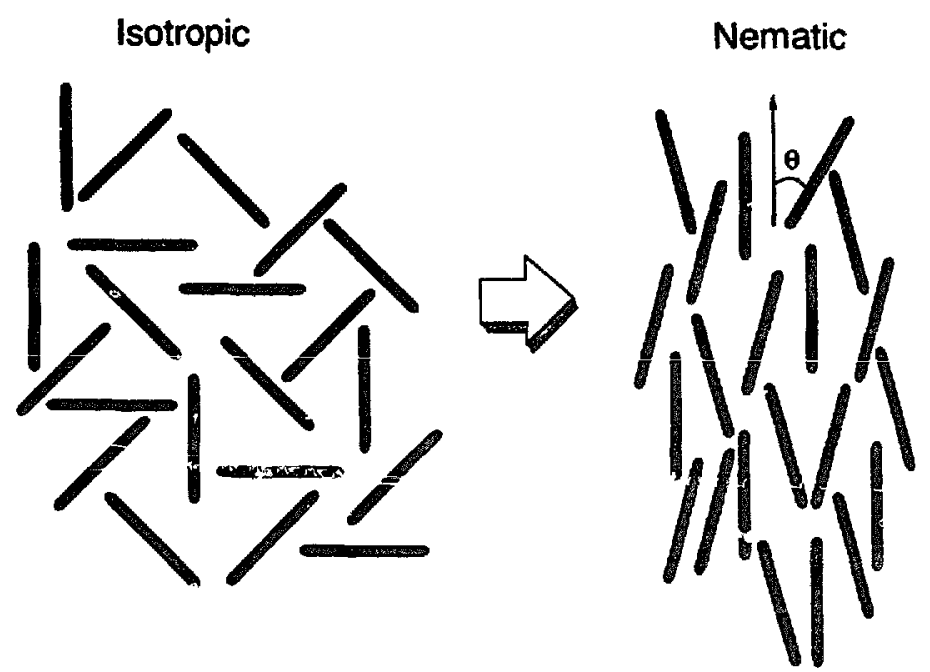

Fig. 5. Schematic representation of the isotropic-nematic transition in a system of hard rods. 
lies at the basis of the explanation for the hard sphere fluid to solid transition. The nematic phase has a lower ideal entropy than the isotropic phase. On the other hand the average excluded volume is smaller in the nematic phase than in the isotropic phase leading to a higher packing entropy in the ordered phase. In fact for very long thin particles the theory for the isotropic-nematic transition becomes particularly simple. Onsager showed that in the limit $L / D \rightarrow \infty$ ( $L$ : length, $D:$ diameter) the dominant effect of excluded volume on the free energy is of order $\rho=N / V$. A quantitative theory of the isotropicnematic transition is therefore possible in the limit of very long thin particles.

Tine volume excluded to a second cylinder due to the presence of the firs: is sketched in fig. 6 and is dependent on the angle $\gamma$ between both cylinders,

$$
v_{\text {excl }} \sim 2 L^{2} D|\sin \gamma| .
$$

In the isotropic phase the orientational average of $|\sin \gamma|$ is $\frac{1}{4} \pi$ and we find

$$
F^{\text {iso }} \sim N k T \rho \frac{1}{4} L^{2} D .
$$

Assuming that in the nematic phase the orientation of the rod-like particles can be described by a Gaussian distribution function

$$
f(\theta) \sim \exp \left(-\frac{1}{2} \alpha \theta^{2}\right)
$$

( $\theta$ : angle between a rod and the director), one finds for sufficiently large values of $\alpha(\alpha \geqslant 20)$

$$
F^{\mathrm{ncm}} \sim N k T\left((\ln \alpha-1)+\rho \frac{4}{\sqrt{\pi \alpha}} \frac{\pi}{4} L^{2} D\right) .
$$

Here the first term on the right hand side describes the increase of the free energy that is caused by the lowering of the ideal entropy. The second term indicates a lowering of the free energy due to the increase of the free volume caused by the orientational ordering of the particles. With increasing number density the latter effect becomes the dominant one and for volume fractions $\rho \pi / 4 L D^{2} \cong 4 D / L$ a transition from the isotopic to the nematic phase takes piace.

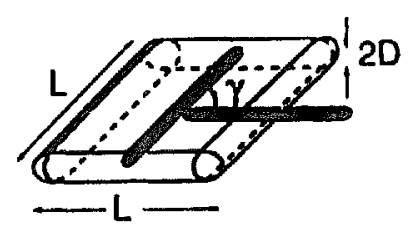

Fig. 6. The excluded volume of two spherocylinders. 
While Onsager concentrated in his theory on the isotropic to nematic iransition he definitely did not exclude the possibility of the occurrence of other types of anisotropic phases in a hard rod fluid such as smectic liquid crystals. Nevertheless it was somehow generally accepted in the literature that smectic liquid crystalline ordering demands that attractive forces also operate. The computer simulations of Frenkel and coworkers [9] however have revealed that hard spherocylinders with full translational and orientational freedom form a thermodynamically stable smectic phase. Again the nematic-smectic phase transition (schematically depicted in fig. 7) can be understood on the basis of a competition between two terms in the entropy. The long range order of the smectic layers reduces the ideal entropy but the more efficient packing of the particles leads to more free volume which in turn leads to a gain in packing entropy. As is clear from the contribution of Frenkel the pirase diagrams of systems of hard-core particles exhibit a remarkable richness of ordering. In particular, nematic, smectic and columnar phases have been observed.

A rigid rod-like particle that has long served as an experimental reference for theoretical studies of orientation order in the systems of hard rods is tobacco mosaic virus (TMV). TMV is a rigid hollow cylinder of length $300 \mathrm{~nm}$ and diameter $18 \mathrm{~nm}$ which carries in the $\mathrm{pH}$ range of 7-8 a negative charge density of about $-5 \mathrm{e} / \mathrm{nm}$. In addition to hard core repulsion TMV particles interact through screened Coulomb rep'lsion which depends on the ionic strength of the solution. In a beautiful series of experiments Meyer and coworkers have made significant contributions to the study of the orientational order in solutions of TMV. By analysis of the angular spread of X-ray diffraction patterns the orientational distribution function is the nematic phase was determined [10]. Good agreement with model calculations based on a

Nematic

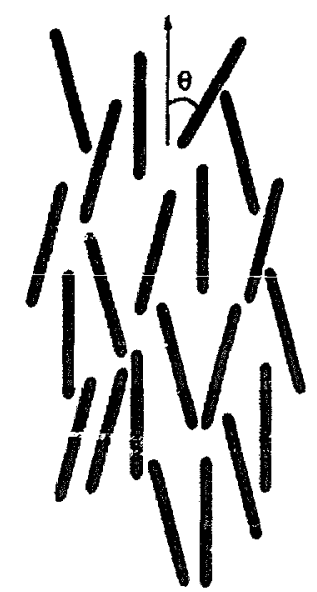

Smectic

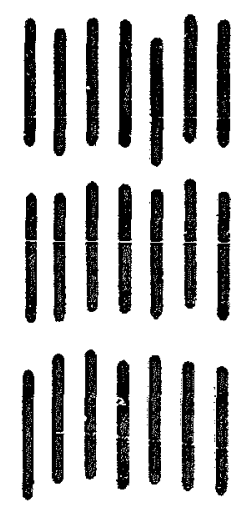

Fig. 7. Schematic representation of th. nematic-smectic transition in a system of hard rods. 
modified Onsager theory of suspensions of hard rods was observed. Also by a combination of optical and X-ray experiments the first definitive experimental evidence of the smectic-A phase in TMV solutions was obtained [11].

\section{Liquid crystal phases in solutions of semi-flexible polymers}

A number of stiff-chain polymers are known to form nematic liquid crystals in solution. Up to now we have considered completely rigid particles. However, in reality stiff polymers always possess at least some degree of flexibility with which we may associate a certain configurational entropy. In the nematic phase all polymers must be more or less parallel to the director which leads to a considerable loss in entropy. An expression for this entropy loss was first derived by Khoklov and Semenov [12] as an extension of the Lifshitz theory [13] for flexible polymers. By introducing the socalled deflection length Odijk [14] has : en able to give a physically appealing picture of this loss of entropy.

Before formulating this deflection length we first consider a semiflexible polymer in dilute solution. Due to the thermal motion the polymer chains will undergo curvature fluctuations leading to deviations from the equilibrium straight shape. The average value of these fluctuations can be calculated from thermodynamic fluctuation theory usit $g$ the curvature elastic free energy as weight factor in the fluctuation probability [15]. The curvature elastic free energy can be written as

$$
F_{\text {elas }}=\frac{1}{2} K \int_{0}^{L}[1 / R(s)]^{2} \mathrm{~d} s,
$$

where $K$ is the bending rigidity and $R(s)$ is the radius of curvature at the point $s$ along the contour of the polymer which has a length $L$. Let $u(s)$ and $u(t)$ be unit tangent vectors at two points $s$ and $t$ along the contour of the chain and let $\theta$ denote the angle between these tangents (see fig. 8). Using const. $\times$ $\exp \left(-F_{\text {ctas }} / k_{13} T\right)$ as probability for curvature fluctuations one obtains [15]

$$
\left\langle\theta^{2}(t-s)\right\rangle \sim|t-s| / P \quad(|t-s| \ll P),
$$

where $P$ is the persistence length which is related to the bending rigidity,

$$
P=K / k_{\mathrm{B}} T \text {. }
$$

In a nematic solution one can imagine each polymer chain to be confined within a virtual tube formed by the topoiogical resirictions due to the surround- 


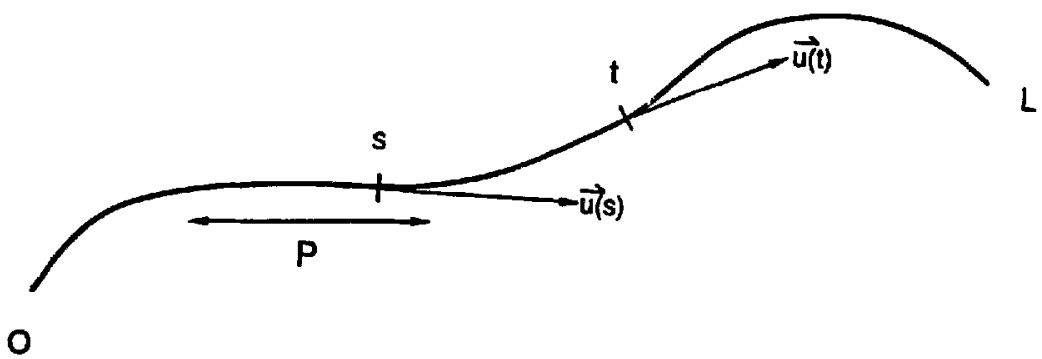

Fig. 8. Semi-flexible chain with an indication of the persistence length $P$.

ing chains. The deflection length $\lambda$ is the distance between collisions in the tube (see fig. 9). The dependence of this length scale on the diameter of the tube $d$ and the persistence length $\boldsymbol{P}$ follows immediately by realizing that in the tube

$$
\left\langle\theta^{2}\right\rangle \sim(d / \lambda)^{2},
$$

whereas application of eq. (6) yields

$$
\left\langle\theta^{2}(\lambda)\right\rangle \sim \lambda / P .
$$

Thus

$$
\lambda^{3} \sim P d^{2} \sim K d^{2} / k_{\mathrm{B}} T
$$

For a nematic phase in which the orientation distribution can be described by a Gaussian, one has [16]

$$
\left\langle\theta^{2}\right\rangle \sim 1 / \alpha
$$

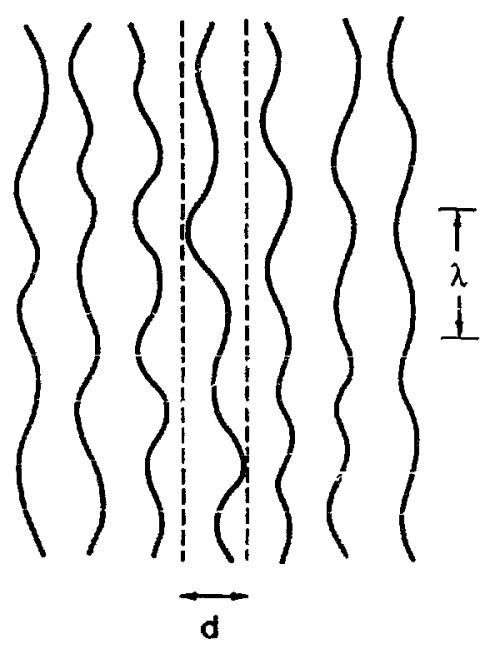

Fig. 9. Schematic representation of a nematic liquid crystal of semi-flexible chains with an indication of the deflection length $\lambda$. 
Combining now eqs. (9) and (11) we obtain

$$
\lambda \sim P / \alpha .
$$

Note that since generally $\alpha \gg 1$ we have $\lambda \ll P$. Using the extensivity requirement for the free energy we find for the increase in free energy resulting from the loss of entropy due the orientational confinement of the polymer chains in the nematic phase the following expression:

$$
\Delta F \sim k_{\mathrm{B}} T(L / \lambda) \sim k_{\mathrm{B}} T(L / P) \alpha .
$$

Roughly speaking the free energy increases of the order $k_{\mathrm{B}} T$ per collision in the confining tube. We see that for a semiflexible chain the increase of the free energy due to orientational confinement is proportional to $\alpha$ instead of $\ln \alpha$ as is the case for rigid rods. Nevertheless above a critical concentration the decrease of the free energy due to the gain in free volume is sufficient to outweigh the above discussed increa. e of the free energy. This means that semiflexible polymers interacting via their mutual excluded volume have a thermodynamically stable nematic phase.

A.gain using the virial expansion of the free energy to order $\rho$ the isotropicnematic transition for semiflexible chains can be worked out quantitatively [17]. In the limit $L \gg P$ the transition now takes place for volume fractions $\frac{1}{4} \rho \pi L D^{2} \cong 5 D / P$. Note that in the limit $L \gg P$ the volume fraction at which the transition occurs is independent of the contour length $L$ i.e. independent of the molecular weight of the polymer.

Teramoto and coworkers have tested the above discussed extension of the Onsager theory with experimental data for phase diagrams and osmotic pressures of carefully prepared solutions of stiff polymers. They showed that the theory is almost quantitative in describing phase diagrams of fairly stiff polymers but fails to describe the data for polymer systems with persistence length $P \leqslant 30 \mathrm{~nm}$. For these systems the critical concentrations are rather high and the failure of the theory appears to be essentially due to the fact that for these cases it is not sufficient to consider only terms up to order $\rho$ in the free energy.

In their contribution Meyer and :oworkers discuss the use of X-ray diffraction to study polymer nematic ordering. They show how irom $\mathrm{X}$-ray diffraction data the orientational distribution function for the polymer chains and an indication of the deflection length can be extracted. These kinds of fundamental information open the way to a deeper understanding of the ordering in these systems.

A particularly interesting example of a stiff chain polymer that shows liquid 
crystallinity in solution is DNA. In fact DNA forms multiple liquid crystalline phases whose nature depends on the polymer concentration. Rill and coworkers have examined in detail the phase behaviour of concentrated solutions of approximately $50 \mathrm{~nm}$ long fragments of double stranded DNA in electrolyte solutions. Part of the motivation for this work on DNA in vitro derives from the fact that DNA packaging in vivo, with (weight) concentrations approaching $70 \%$ in sperm heads and virus capsids may be related to this natural tendency of DNA to form liquid crystalline phases in concentrated solutions. Livolant in her contribution prevides a detailed comparison between the different liquid crystailine phases of DNA observed in vitro when increasing the polymer concentration and the different forms of condensed chromatin in vivo.

Another area where polymer liquid crystals play an important role is in the processing of high strength, high modulus fibres such as the widely known aramid polymer PPTA poly(p-phenyleneterephtalamide) also commercially known as Twaron and Kevlar. Achievement of the ultimate properties of the

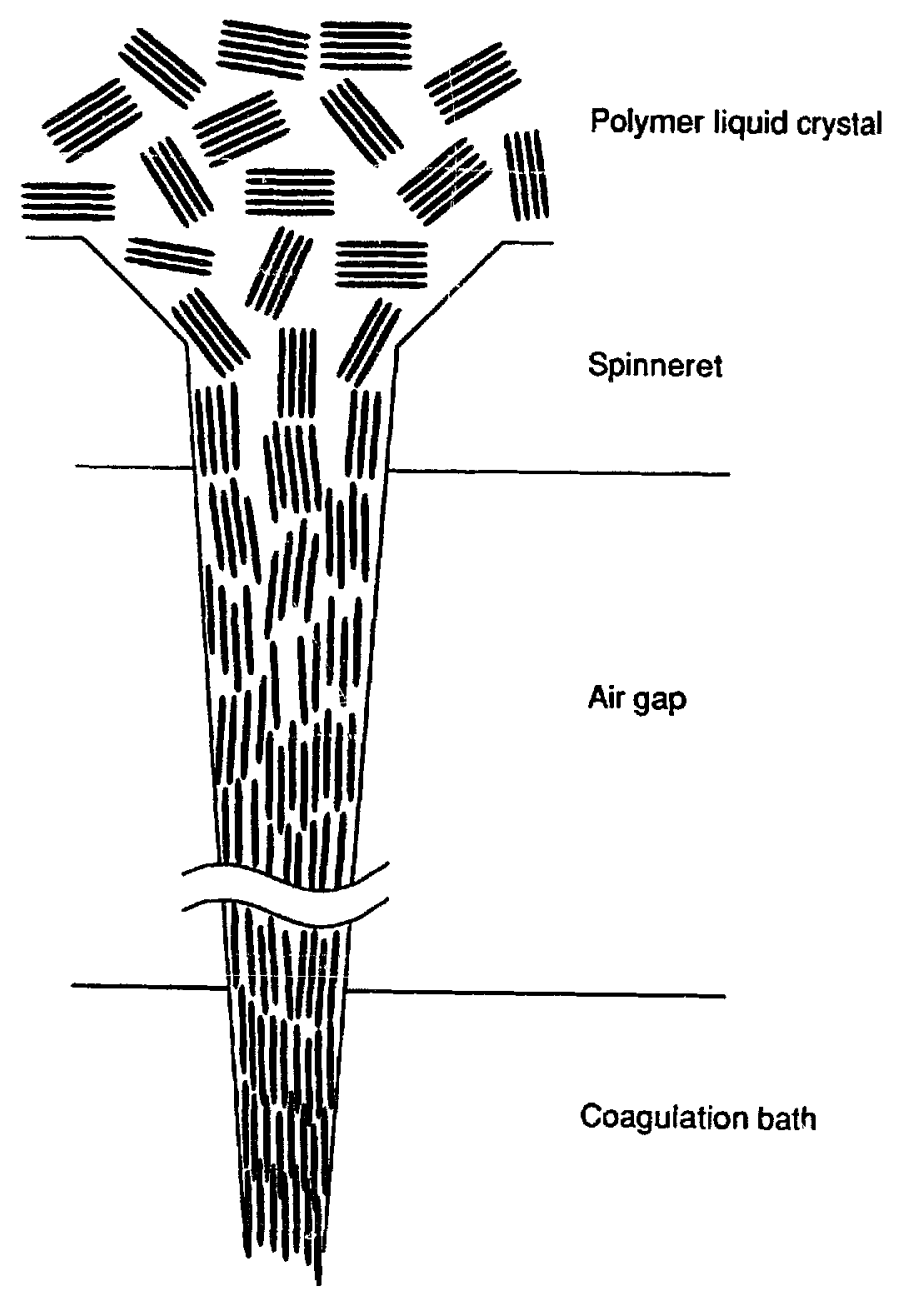

Fig. 10. Schematic drawing of the wet spinning process. 
fibres depends upon the achievement of a high degree of orientational order of the polymer chains in the spinning process. Flow induced order plays an important role here. During the spinning process the local directors are aligned by the shear flow in the capillaries in the spinneret and by the elongational fiow in the entrance zone above the spinneret and in the air gap (see fig. 10). Odell in his contribution describes interesting fundamental work on shear induced order in solutions of rigid polymers that could well lead to an improved understanding of the spinning process.

\section{Liquid crystalline phases in surfactant systems}

Surfactants (shorthand for surface active agents) are amphiphilic molecules which contain a hydrophilic head group and a hydrophobic hydrocarbon tail (or tails). Due to their amphiphilic nature, surfactants have a remarkable tendency to aggregate in a variety of forms whose exact structures depend on some simple, geometric packing considerations [18]. These aggregates may adopt an ordered structure and form liquid crystalline phases. Three classes of amphiphilic liquid crystalline phases have been recognized [19], these being lamellar, hexagonal and cubir. Whereas the lamellar and hexagonal phase structures are well established the complete determination of the cubic structures remains one of the most important problems yet to be solved [20]. In fact one can distinguish two types of cubic phases. On the one hand there are "micellar" cubic phases that consist of cubic close packed arrays of small globular micelles. On the other hand there are "bicontinuous" cubic phases having microstructures which exhibit simultaneous continuity of hydrophilic and hydrophobic components. The general phase diagram of liquid crystalline phases built by amphiphilic moleculars is shown in fig. 11. A remarkable feature is that similar sequences of structures occur rather independent of the chemical details of the molecules. Tiddy gives an explanation of this scquence in terms of a balance between micelle-micelle interactions and intramicelle forces whereas Carvolin considers amphiphilic liquid crystalline phases in terms of pericdic systems of frustrated interfacial films. This analysis leads indeed to a sequence of structures similar to the observed one.

Anderson discusses recently discovered processes in which one or more components of an amphiphilic liquid crystal is polymerized in such a way as to preserve and fixate the microstructures. Of particular importance are the bicontinuous cubic phases where polymerization of the amphiphilic component followed by removal of the remaining liquid component leads to extremely well defined microporous materials. There appear to be several areas of potential application for these novel materials. 


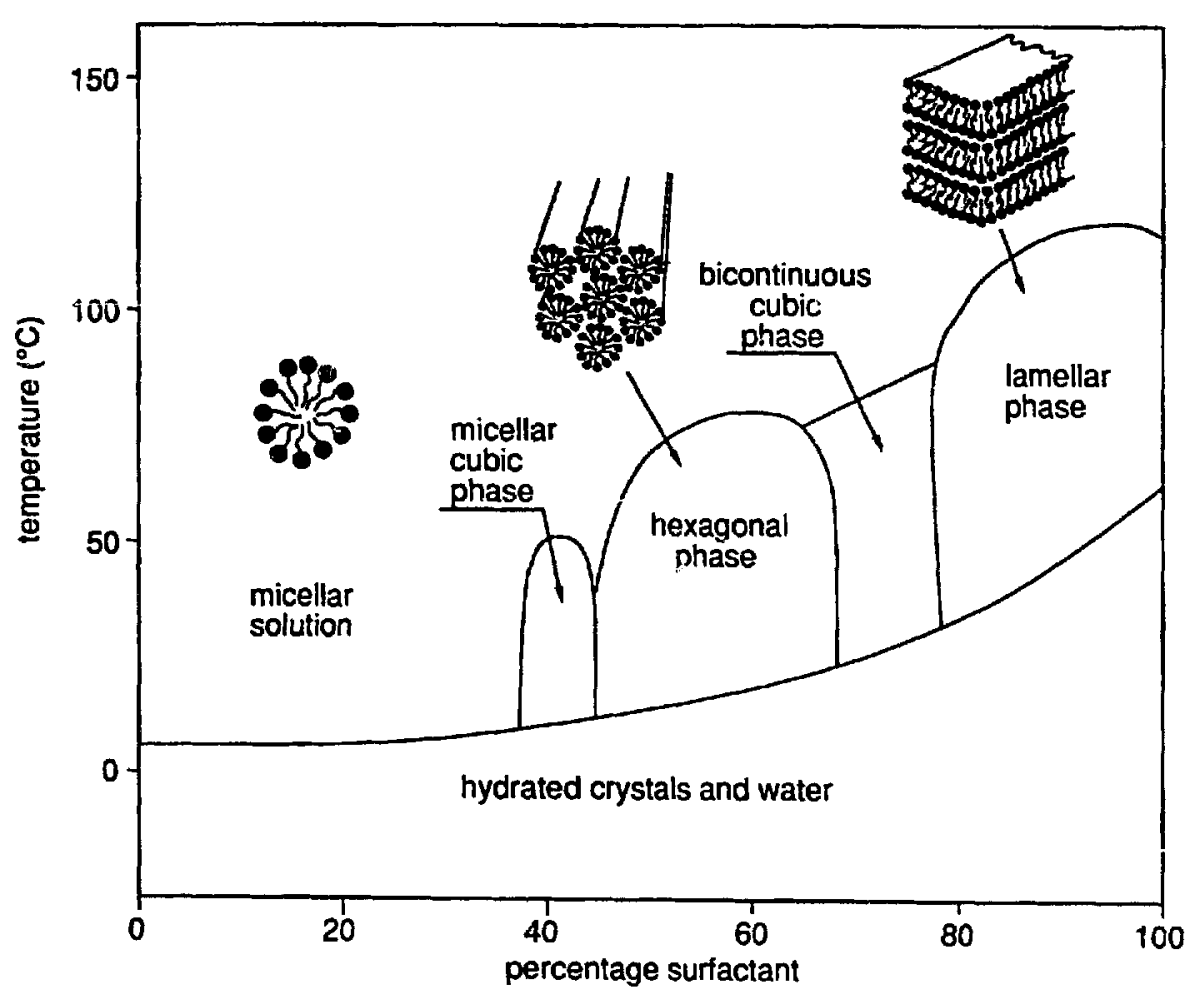

Fig. 11. Typical surfactant-water phase diagram.

Most amphiphilic lamellar liquid crystalline phases $\left(L_{\alpha}\right)$ show restricted swelling. This restricted swelling is due to the fact that attractive van der Waals interactions prevent the amphiphilic bilayers from separating more than 2$3 \mathrm{~nm}$. In recent years however it was discovered that in a number of surfactant systems the $L_{\alpha}$ phase can be swollen by water almost without bound leading to bilayer distances as large as hundreds of nanometers [21-24]. Light scattering [23] and X-ray scattering [22] show well defined Bragg peaks providing strong evidence of true lamellar order. These hyper-swollen lamellar liquid crystals are found in binary systems containing a non-ionic surfactant (such as $C_{12} E_{5}$ ) and water and in quaternary systems containing an ionic surfactant (such a: SDS), a long-chain alcohol (such as pentanol) and water with salt. The common feature appears to be that the amphiphilic bilayers in these systems have a very small bending rigidity. Due to the small bending rigidity the bilayers undergo strong thermal undulations. The mutual hindrance of the undulating bilayers gives rise to a steric repulsion of entropic origin. This steric repulsion appears to be able to overcome the attractive interactions leading to an "unbinding" of the lamellae.

There is a very close analogy between the confinement entropy of semiflexible bilayers in the $L_{\alpha}$ phase and the confinement entropy of semiflexible chains in a polymer nematic liquid crystal. Assuming that there is no spontaneous 
curvature, the curvature elastic free energy of a semiflexible bilayer can be written as [25]

$$
F_{\text {elas }}=\int\left[\frac{1}{2} K\left(1 / R_{1}+1 / R_{2}\right)^{2}+\bar{K} / R_{1} R_{2}\right] \mathrm{d} A .
$$

Here $R_{1}$ and $R_{2}$ are the local principal radii of curvature and $K$ and $\bar{K}$ are the elastic bending moduli of mean and Gaussian curvatures respectively. Let $\boldsymbol{n}(s)$ and $n(t)$ be normals at two points $s$ and $t$ on the surface and let $\theta$ denote the angle between these normals (see fig. 12). Using const. $\times \exp .\left(-F_{\text {elas }} / k_{\mathrm{B}} T\right)$ as probability for curvature fluctuations one obtains [26]

$$
\left\langle\theta^{2}(t-s)\right\rangle \cong\left(\pi K / k_{\mathrm{B}} T\right) \ln (|t-s| / a) .
$$

Here $a$ is a short distance cutoff of about the size of an amphiphilic moleculer that must be introduced in order to avoid a divergence. Eq. (15) leads to a persistence length

$$
P \cong a \exp \left(2 \pi K / k_{\mathrm{B}} T\right)
$$

For most amphiphilic bilayers $K \gg k_{\mathrm{B}} T$ (say $10 k_{\mathrm{B}} T$ or more) and thus they are flat over macroscopic distances. However in the surfactant systems that exhibit highly swollen lamellar phases the bilayers have a very low rigidity $K \cong k_{\mathrm{B}} T$ and thus $P \sim 100 \mathrm{~nm}$.

We now return to the rolc of the low rigidity in understanding the stability of swollen $\mathrm{L}_{\alpha}$ phases. In these phases the thermal und 'iations are hindered by collisions with the neighbouring bilayers (see fig. 13). Like in the case of semiflexible chains confined in a tube we can also here formulate the deflection

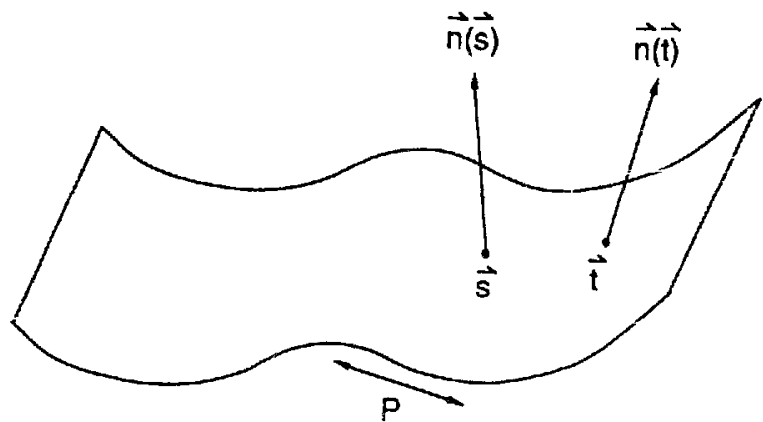

Fig. 12 .

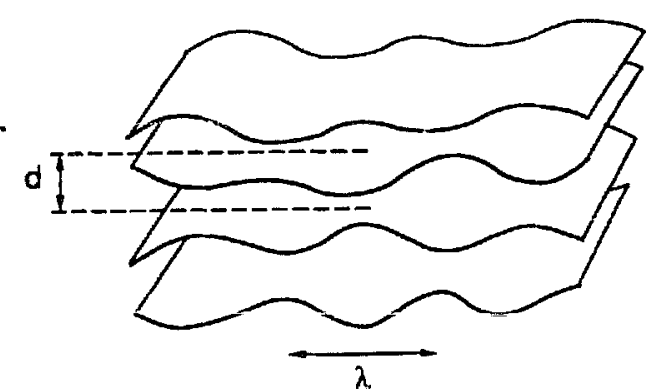

Fig. 13 .

Fig. 12. Semi-flexible surface with an indication of the persistence length $P$.

Fig. 13. Lamellar phase of semi-flexible surfaces with an indication of the deflection length $\lambda$. 
lergth $\lambda$ as the distance between collisions. The dependence of this scale on the width of the gap $d$ and the bending rigidity $K$ follows immediately by realizing that in the gap

$$
\left\langle\theta^{2}\right\rangle \sim(d / \lambda)^{2}
$$

Furthermore for $d<P$ the logarithmic term in (5) for distances between collisions will be of order unity and we may write

$$
\left\langle\theta^{2}(\lambda)\right\rangle \sim K / k_{\mathrm{B}} T
$$

Thus

$$
\lambda^{2} \sim K d^{2} / k_{\mathrm{B}} T
$$

The difference in dimensionality between the semi-flexible chain and the semi-flexible surface problem is reflected in the different powers of $\lambda$ occurring in eqs. (10) and (19) respectively. Using the extensivity requirement we find for the confinement free energy

$$
\Delta F \sim k_{\mathrm{B}} T A / \lambda^{2} \sim\left[\left(k_{\mathrm{B}} T\right)^{2} / K d^{2}\right] A
$$

where $A$ is the area of the bilayer $\left(A \gg \lambda^{2}\right)$. This confinement free energy of flexible bilayers was first introduced and evaluated by Helfrich [27]. There is strong evidence that the steric repulsion to which this confinement free energy gives rise dominates the interbilayer interaction in swollen $L_{\alpha}$ phases $[22,23]$.

A first order transition between the swollen $\mathrm{L}_{\alpha}$ phase and an isotropic phase of random bilayers has been observed. The latter phase is referred to as the $L_{3}$ phase. Although isotropic, it is strongly birefringent. The microstructure of the $L_{3}$ phase and the statistical mechanical treatment of the $L_{3}$ to $L_{\alpha}$ transition in dilute surfactant system is discussed in the contributions by Porte and Cates. There are issues that are not completely resolved (like for instance the (in)stability of a nematic phase of semiflexible bilayers between the $\mathrm{L}_{3}$ and $\mathrm{L}_{\alpha}$ phase) [28], which require a fuller understanding of the statistical mechanics of semi-flexible random surfaces.

\section{References}

[1] A.K. van Helden, J.W. Jansen and A. Vrij, J. Coll. Interf. Sci. 81 (1981) 354.

[2] L. Antl, J.W. Goodwin, R.D. Hill, R.H. Ottewill, S.W. Owens and S. Papworth, Coll. Surf. 17 (1986) 67. 
[3] B.J. Alder and T.E. Wainwright, J. Chem. Phys. 27 (1957) 1208.

[4] W.G. Hoover and F.H. Ree, J. Chem. Phys. 49 (1968) 3609.

[5] D. Oxtoby, Nature 347 (1990) 725; M. Baus, J. Phys. Condens. Matter 2 (1990) 2111.

[6] B.J.A. Ackersonin, Physica A 174 (1991) 15.

[7] H. Zocher, Z. Anorg. Chem. 147 (1925) 91.

[8] L. Onsager, Phys. Rev. 62 (1942) 558; Ann. N.Y. Acad. Sci. 51 (1949) 627.

[9] D. Frenkel, H.N.W. Lekkerkerker and A. Stroobants, Nature 332 (1988) 822.

[10] R. Oldenbourg, X. Wen, R.B. Meyer and D.L.D. Caspar, Phys. Rev. Lett. 61 (1988) 1851.

[11] X. Wen, R.B. Meyer and D.L.D. Caspar, Phys. Rev. Lett. 63 (1989) 2760.

[12] A.R. Khoklov and A.N. Semenov, Physica A 108 (1981) 546.

[13] I.M. Lifshitz, Sov. Phys. JETP 28 (1969) 1280.

[14] Th. Odijk, Macromolecules 16 (1983) 1340.

[15] L.D. Landau and E.M. Lifshitz, Statistical Physics, 3rd Ed., part 1 (Pergamon, Oxford, 1980) pp. 396-400.

[16] Th. Odijk, Macromolecules 19 (1986) 2313.

[17] G.J. Vroege and Th. Odijk, Macromolecules 21 (1988) 2848.

[18] J.N. Israelachvili, D.J. Mitchell and B.W. Ninham, J. Chem. Soc. Faraday Trans. II, 72 (1976) 1525.

[19] P. Ekwall, in: Advances in Liquid Crystals, G.H. Brown, ed., vol. 1 (Academic Press, New York, 1971) ch. 1.

[20] G.J.T. Tiddy, Phys. Rep. 57 (1980) 1.

[21] W.J. Benton and C.A. Miller, J. Phys. Chem. 87 (1983) 4981.

[22] C.R. Safinya, D. Roux, G.S. Smith, S.K. Sinha, F. Dimon, N.A. Clark and A.M. Bellocq, Phys. Rev. Lett. 57 (1986) 2718.

[23] F.C. Larche, J. Appell, G. Porte, P. Basserau and J. Marignan, Phys. Rev. Lett. 56 (1986) 1700.

[24] R. Strey, R. Schomäcker, D. Roux, F. Nallet and U. Olsson, J. Chem. Soc. Faraday Trans. 86 (1990) 2253.

[25] W. Heifrich, Z. Naturforsch. 28C (1973) 693.

[26] P.G. de Gennes and C. Taupin, J. Phys. Chem. 86 (1982) 2294.

[27] W. Helfrich, Z. Naturforsch. 33A (1978) 305.

[28] Th. Odijk, J. Chem. Phys. 88 (1988) 7167. 